\title{
Malignant Peripheral Nerve Sheath Tumour in a Patient of Type 1 Neurofibromatosis
}

\author{
AMITAVA SENGUPTA, ${ }^{1}$ ANKAN BANDYOPADHYAY, ${ }^{2}$ DEBRAJ JASH,${ }^{3} \mathrm{KAUSHIK} \mathrm{SAHA}^{4}$
}

\begin{abstract}
:
Malignant peripheral nerve sheath tumours (MPNST) constitute a rare type of soft tissue sarcoma which arises from peripheral nerves or Schwann cells, perineural cells or fibroblasts. MPNST is a rare neoplasm affecting $I$ in I,00,000 population. Here we report a case of a 36 year old male who presented to us with an upper abdominal swelling for last 3 months. At first, a diagnosis of type I neurofibromatosis type I (NF-I) was made. Contrast enhanced CT scan abdomen and thorax revealed presence of a complex, heterogenous mass in upper abdomen with extension towards left hemithorax. Histopathological examination done from true cut biopsy specimen of the mass was suggestive of malignant peripheral nerve sheath tumour. Positivity of tumour cells on immunohistochemistry to S-100 established the diagnosis of MPNST in type I neurofibromatosis. In conclusion, it was a rare case of MPNST in a patient of NF-I.
\end{abstract}

Keyword: Malignant peripheral nerve sheath tumour, Neurofibromatosis type I, Upper abdominal mass

\section{Introduction:}

Malignant peripheral nerve sheath tumours (MPNST) are sarcoma which arises from peripheral nerves or from cells associated with nerve sheath. In general, sarcoma arising from peripheral nerve or neurofibroma is considered to be a MPNST. The incidence of malignant peripheral nerve sheath tumour (MPNST) in general population is $0.001 \%$. Its incidence in patients with Neurofibromatosis type 1 (NF1) is $4.6 \%$. Here we present a rare case of MPNST which extended to thorax after originating inside the abdomen in a patient of NF1.

\section{Case report:}

A 36 year old male presented with pain and upper abdominal swelling for last 3 months along with heaviness of chest for last 2 months. On general survey, there was presence of multiple cafe au lait macules $2 \mathrm{~cm}$ in diameter all over the trunk. Patient also had numerous neurofibroforma all over

1. Associate Professor, Department of Pulmonary Medicine, NRS Medical College and Hospital, West Bengal, India

2. RMO cum Clinical Tutor, Department of Pulmonary Medicine, NRS Medical College and Hospital, West Bengal, India

3. Post Graduate Trainee, Department of Pulmonary Medicine, NRS Medical College and Hospital West Bengal, India

4. RMO cum Clinical Tutor, Department of Pulmonary Medicine, NRS Medical College and Hospital,West Bengal, India

Correspondence : Dr. Kaushik Saha ,Rabindra Pally, $1^{\text {st }}$ Lane, P.O. - Nimta, Kolkata - 700049, West Bengal, India , e mail doctorkaushiksaha@gmail.com the body with axillary and inguinal freckles. So, a diagnosis of type 1 neurofibromatosis (NF-1) was made. Examination findings of respiratory tract were suggestive of left sided pleural effusion. On abdominal examination a tender, firm to hard $(10 \mathrm{~cm} \times 8 \mathrm{~cm})$ epigastric swelling was noted with fixity to underlying structure and overlying skin. Cardiovascular and neurological examination were within normal limit. Contrast enhanced CT scan of abdomen and thorax showed a large $(11 \mathrm{~cm} 17 \mathrm{~cm})$ complex heterogenous upper abdominal mass with upward extension towards left hemithorax along the paracardiac region in the left lung up to the horizontal level of main pulmonary artery (Figure 1).

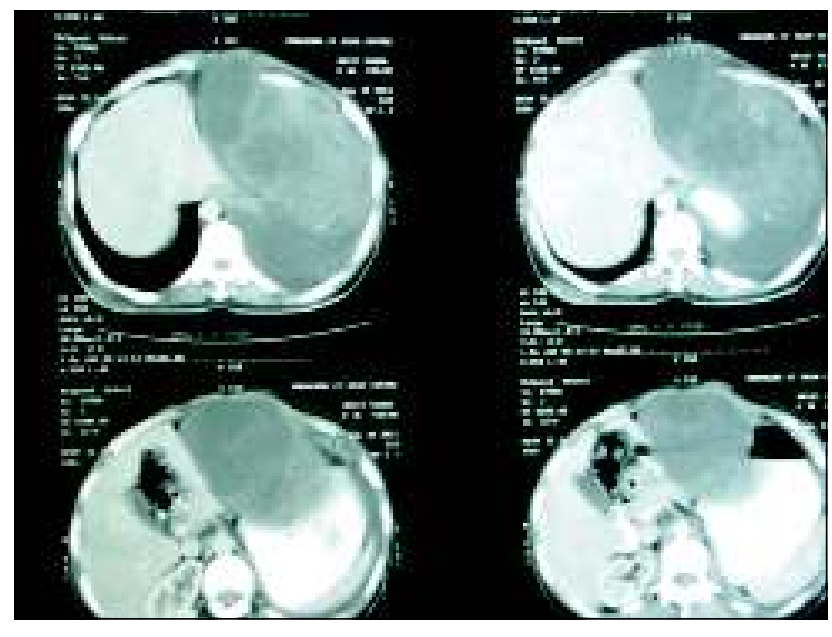

Fig.-1: Contrast enhanced CT scan of abdomen showing a large $(11 \mathrm{~cm} \times 17 \mathrm{~cm})$ complex heterogenous upper abdominal mass. 
There was also presence of massive left sided pleural effusion with lung mass. (Figure 2).

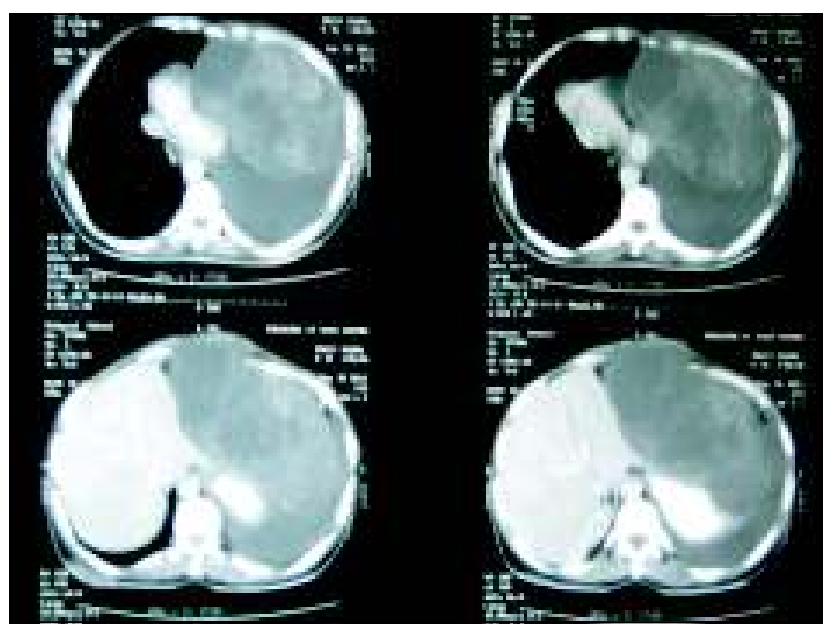

Fig.-2: CT scan thorax with contrast showing left lung mass with pleural effusion.

CT guided fine needle aspiration cytology (FNAC) from the abdominal mass showed fat globules, necrosed materials and pleomorphic spindle cells. Histopathological examination from the trucut biopsy specimen from the mass showed interlacing fascicles of spindle shaped to oval cells with vesicular nuclei. Tumour cells demonstrated moderate nuclear pleomorphism and exhibit brisk mitotic activity (mitotic count $>6 / 10 \mathrm{HPF}$ ) (Figure 3 ).
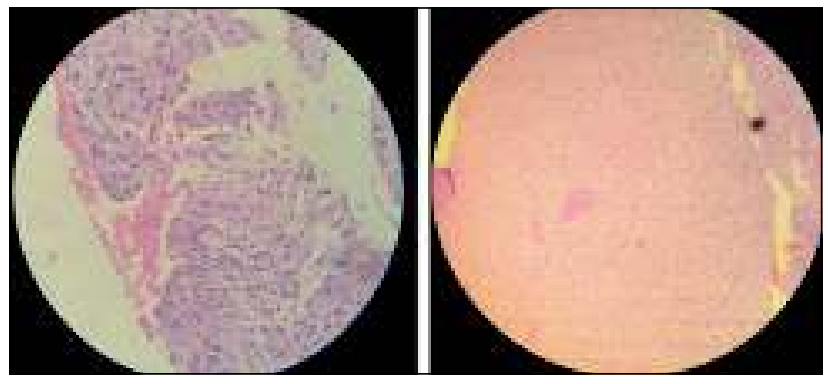

Fig.-3: Biopsy specimen showing interlacing fascicles of spindle shaped to oval cells with vesicular nuclei and demonstrating moderate nuclear pleomorphism with brisk mitotic activity.

Plenty of blood vessels with thrombus within it and areas of necrosis was seen. On immunohistochemistry, the tumour cell was S100 positive, immunonegative to c-kit and CD34. Mib levelling index was $70 \%$. In context of history of NF-1, the above histopathological picture was suggestive of MPNST. CT scan and MRI of brain did not reveal any abnormality. On thoracotomy, the mass was fixed to the diaphragm and parietal wall with extension to the abdomen. So, resection of the tumour could not be done. Patient received chemotherapy with doxorubicin, cyclophosphamide and vincristin for 2 cycles after which he died subsequently.

\section{Discussion:}

NF-1 is an autosomal dominant genetic disorder with a prevalence of approximately 1 in 4,000 births and no racial predilection. ${ }^{1} \mathrm{NF}-1$ is diagnosed by any two of the six criteria (1) Multiple cafe-au-lait macules larger than $1.5 \mathrm{~cm}$ in diameter and more than six in number. (2) Axillary or inguinal freckles, Two or more neurofibromas of any type or one plexiform neurofibroma, (3) Sphenoid wing dysplasia or congenital bowing of long bone cortex with or without pseudoarthrosis, (4) Bilateral optic nerve glioma, two or more Lisch nodules on slit lamp examination, (5) First degree relative with NF1 diagnosed by preceding criteria.

A sarcoma is defined as MPNST if at least two of the following criteria are met with. (1) It arises from a peripheral nerve, (2) It arises from a pre-existing benign nerve sheath tumour (neurofibroma), (3) It demonstrates Schwann cell differentiation on histological examination. The incidence of MPNST in general population is $0.001 \%$. Its incidence in patients with NF1 is $4.6 \%{ }^{2} \mathrm{Up}$ to $50 \%$ of MPNST occurring in patients with NF1 arises from a pre-existing neurofibroma. ${ }^{3}$ Overall NF1 patients have a $4 \%$ lifetime risk of ultimately developing a MPNST. ${ }^{4}$ MPNST in patients of NF-1 generally occurs in adulthood, median age being 30 years only. ${ }^{5}$ MPNSTs most commonly occur in the deep soft tissues, usually close to a nerve trunk. The most common sites are the sciatic nerve, brachial plexus, and sacral plexus. It usually presents as an enlarging palpable mass. Pain is a variable complaint. Rapid enlargement occurs more often in patients of NF1. Neurofibromas typically have low attenuation on CT, which may be related to the fat content of myelin from Schwann cells, the high water content of myxoid tissue, entrapment of fat, and cystic areas of hemorrhage and necrosis. ${ }^{6}$ MPNST arising from a peripheral nerve may result in variable clinical patterns including radicular pain, paresthesia and motor weakness of muscles. Haematogenous metastasis from MPNST occurs most commonly in lungs. ${ }^{7}$

S-100 is traditionally regarded as the best marker for malignant peripheral nerve sheath tumour; however, it is positive in only about one third to half the tumors. ${ }^{8}$ The negative reaction may be caused by not only tumour cell anaplasia but also by different constituents other than Schwann cell. All perineural MPNST were S100 protein negative and EMA and/or GLUT1 positive. For the immunohistochemical diagnosis of MPNST, nerve sheath markers other than S100 protein such as CD57 (Leu7), EMA, Glut1, CD34 should also be included in the panel of antibodies. ${ }^{9}$ 
The reported five year survival for patients with MPNST without NF1 is as high as $50 \%$.It drops down to as low as $10 \%$ in MPNST patients with NF1. ${ }^{7}$

Treatment consists of complete and wide surgical resection. ${ }^{10}$ Adjuvant irradiation seems to improve local control of the disease. Use of chemotherapy is only employed in high grade disease in which metastatic disease is likely. Chemotherapy can be administered in preoperative or postoperative settings. The prognosis depends on tumour size, surgical excision margin, and histological presence of necrosis and history of prior irradiation. ${ }^{8}$. MPNST itself is a rare disease. Moreover MPNST being associated with NF-1 was more uncommon. Initial Presentation having abdominal mass with extension into the lung made the case more atypical. Patient did not respond to chemotherapy as he fell into bad prognosis group due to presence of large size tumour, failure of surgical resection and presence of necrosis in the histopathological specimen.

\section{Conflict of Interest : None}

\section{References:}

1. Mulvihill JJ. Malignancy: epidemiologically associated cancers. In: Huson SM, Hughes RAC, eds. The neurofibromatoses: a pathogenetic and clinical overview. Cambridge, England: Cambridge University Press, 1994; 305-15

2. Ducatman BS, Scheithauer BW, Piepqras DG, Reiman HM. Malignant peripheral nerve sheath tumors in childhood. J Neurooncol, 1984; 2(3): 241-8

3. Wick MR, Swanson PE, Scheithauser BW, Manivel JC. Malignant peripheral nerve sheath tumor: an immunohistochemical study of 62 cases. Am J Clin Pathol 1987; 87:425-33

4. Meis JM, Enzinger FM, Martz KL, et al. Malignant peripheral nerve sheath tumor (malignant schwannoma) in children.Am J Surg Pathol 1992; 16:694-707

5. Ramanathan RC,Thomas JM Malignant peripheral nerve sheath tumours associated with von Recklinghausen's neurofibromatosis. Eur J Surg Oncol 1999;25:190-3

6. Murphey MD, Smith WS, Smith SE, Kransdorf MJ, Temple HT. Imaging of musculoskeletal neurogenic tumors: radiologic-pathologic correlation. Radio Graphics 1999; 19:1253-80

7. Doorn PF, Molenaar WM, Buter J, Hoekstra HJ. Malignant peripheral nerve sheath tumors in patients with and without neurofibromatosis. Eur J Surg Oncol 1995; 21: 78-82

8. Johnson MD, Glick AD, Davis BW. Immunohistochemical evaluation of Leu-7, myelin basic protein, S-100 protein, glial fibrillary acidic protein, and LN3 immunoreactivity in nerve sheath tumors and sarcomas. Arch Pathol Lab Med1988; 112:155-60

9. Zhou H, Coffin CM, Perkins SL, Tripp SR, Liew M, Viskochil DH. Malignant peripheral nerve sheath tumor: a comparison of grade, immunophenotype, and cell cycle/ growth activation marker expression in sporadic and neurofibromatosis 1-related lesions. Am J Surg Pathol 2003;27:1337-45

10. Ferner RE, Gutmann DH. International consensus statement on malignant peripheral nerve sheath tumours in neurofibromatosis. Cancer Res 2002; 62:1573-77 